Voix et Images

voixetimages

\title{
Bibliographie de Roland Giguère
}

\section{Roger Chamberland}

Volume 9, numéro 2, hiver 1984

Roland Giguère

URI : https://id.erudit.org/iderudit/200440ar

DOI : https://doi.org/10.7202/200440ar

Aller au sommaire du numéro

Éditeur(s)

Université du Québec à Montréal

ISSN

0318-9201 (imprimé)

1705-933X (numérique)

Découvrir la revue

Citer ce document

Chamberland, R. (1984). Bibliographie de Roland Giguère. Voix et Images, 9(2),

75-89. https://doi.org/10.7202/200440ar d'utilisation que vous pouvez consulter en ligne.

https://apropos.erudit.org/fr/usagers/politique-dutilisation/ 


\title{
Bibliographie de Roland Giguère
}

\author{
par Roger Chamberland, Université Laval
}

\begin{abstract}
Note: Cette bibliographie ne contient que les articles portant sur l'oeuvre écrite de Roland Giguère. Exceptionnellement, nous avons inclus quelques textes traitant des rapports entre sa production plcturale et sa production écrite.
\end{abstract}

\section{OEUVRES}

Faire naître. Illustrations d'Albert Dumouchel, Montréal, (les Editions Erta), 1949, (38) f.

Les Nuits abat-jour. Poèmes, avec images d'Albert Dumouchel, (Montréal), Éditions Erta, (1950), (48) p.; reproduit en partie dans l'Âge de la parole. Poèmes 1949-1960, 170 p. (v.p. 63-74).

3 pas. Gravures de Conrad Tremblay, (Montréal), les Éditions Erta, (1950), (13) $\mathrm{p}$.

Avec Théodore Koenig. Le Poème mobile. Poème écrit en collaboration par Théodore Koenig et Roland Giguère le 25 mai 1950 sur une distance de 362 milles Boston-Montréal, (Montréal), les Éditions Erta, 1950, (7) f.

Midi perdu. Dessins de Gérard Tremblay, Montréal, Éditions Erta, 1951, 8 p.; dans l'Âge de la parole. Poèmes 1949-1960, 170 p. (v.p. 77-82).

Yeux fixes ou l'Ébullition de l'intérieur. (Montréal), Editions Erta, (1951), (2) 18 p.; dans l'Âge de la parole. Poèmes 1949-1960, 170 p. (v.p. 85-101), et dans Jean Fisette, le Texte automatiste, essai de théorie pratique de sémiotique textuelle, Montréal, les Presses de l'Université du Québec, 1977, XXIV, 181 (1) p. (v.p. 173-181).

Images apprivoisées. (Clichés de l'auteur), Montréal, Éditions Erta, (1953), (35) $\mathrm{p}$.

Les Armes blanches. Avec 6 dessins de l'auteur, (Montréal), Éditions Erta, (1954), (25) f. (Collection la Tête armée); dans l'Âge de la parole. Poèmes 1949-1960, 170 p. (v.p. 103-121).

Eight Poems from «les Armes blanches», translated by Jean Beaupré and Gael Turnbull, Toronto, Contact Press, (1955, 24 p.). 
Le Défaut des ruines est d'avoir des habitants. (Montréal), Éditions Erta, 1957, 107 p. (Illustré de dessins de l'auteur). «Lieux exemplaires» parut dans Écrits du Canada français, vol. III, 1957, p. 119-134. (Certains extraits furent repris dans:) l'Âge de la parole. Poèmes 1949-1960, 170 p. (v.p. 123-138, 141-147); dans la Main au feu, 1949-1968, 145 p. (v.p. 13-29, 31-43, 70). «Grimoires» parut dans Forêt vierge folle, 219 p. (v.p. 8-83). «Miror" and "Letters to an Escapee», translated by Sheila Fischman, (Erin (Ontario), Porcepic Press, 1977, n.p.).

Adorable Femme des neiges. Poème illustré par l'auteur, Châteaunoir (Aixen-Provence), Erta, (1959, 12 feuillets sous étui); suivi du «Dialogue entre l'Éphémère et l'Immobile», Écrits du Canada français, vol. XVI, 1963, p. 109-128; dans l'Áge de la parole. Poèmes 1949-1960, 170 p. (v.p. 151-162).

L'Âge de la parole. Poèmes 1949-1960. (Montréal), Éditions de l'Hexagone, (1965), $170 \mathrm{p}$. (Collection Rétrospectives). La majorité des poèmes ont été traduits en italien par Novella Novelli sous le titre l'Età della parola, Roma, Bulzoni editore, 1983, $156 \mathrm{p}$.

Pouvoir du noir. Préface de Gilles Hénault, Montréal, Ministère des Affaires culturelles, Musée d'art contemporain, 1966, (n.p.). (Illustrations de l'auteur); dans la Main au feu, 1949-1968, 145 p. (v.p. 113-123).

12 dessins-drawings. (Montréal), Graph, (1966), 12 planches.

Naturellement. Poésie, (Montréal), Éditions Erta, (1968), (8 feuillets illustrés de 8 sérigraphie de l'auteur); dans la Main au feu, 1949-1968, 145 p. (v.p. 125-134).

La Main au feu, 1949-1968. (Montréal), Éditions de l'Hexagone, (1973), $145 \mathrm{p}$. (Collection Rétrospectives).

Édition de luxe avec une sérigraphie de l'auteur sous boitier en raku réalisé par Monique B. Ferron. Reliure et lettrage Simone B. Roy.

La Sérigraphie d̀ la colle. Montréal, Éditions Formart, 1973, 32 p.

Abécédaire. Illustrations de Gérard Tremblay. (Montréal, Édition Erta, 1975). 1 emboitage.

J'imagine. Avec dix lithographies de Gérard Tremblay, (Montréal, Éditions Erta, 1976, 10 f.

Forêt vierge folle. Montréal, l'Hexagone, 1978, 219 p. (Collection Parcours).

10 Cartes postales. (Montréal), Éditions Aubes 3935, 1982.

À l'orée de l'oeil. Cinquante dessins accompagnés d'un texte de Gilles Hénault, (Saint-Lambert), Éditions du Norôit, (1981), 109-(7)p. 


\section{ÉTUDES GÉNÉRALES}

(Anonyme), "Prix littéraire à Roland Giguère», le Droit, vol. 54, no 11 (9 avril 1966), p. 11.

"De l'âge de la parole à l'âge de l'image», la Presse, vol. LXXXII, no 88 (16 avril 1966), p. 6.

, «Black Power», Time (Canadian edition), vol. 88, no 15 (october 7, 1966), p. 17-18.

, «Décerné à Paris. Le prix France-Canada à Roland Giguère», le Devoir, vo. LVII, no 274 (25 novembre 1966), p. 12.

1969), p. 6-11.

, "Quatre poètes et la poésie», Culture vivante, no 12 (février

, «Fiche J.L.Q. Roland Giguère», Vient de paraître, vol. 5, no 1 (février 1969), p. 34.

, «Roland Giguère: profil d'une démarche surréaliste», Vie des arts, vol. 20, no 80 (automne 1975), p. 42-46.

, «Les Prix du Québec 1982. Ceux-là dont les oeuvres sont bien souvent au coeur de nos relations avec les autres...», le Soleil, vol. LXXXVI, no 280 (27 novembre 1982), p. D-2.

ALMERAS, Diane, «Folie de poète», Relations, no 465 (décembre 1980), p. 343.

AYLWIN, Ulric, «Introduction à l'âge de Roland Giguère», la Barre du jour, nos 11-13 (décembre 1967-mai 1968), p. 11-20.

AYRE, Robert, «Painters work being shown in two local exhibitions. Art notes." The Montreal Star, vol. XCII, no 67 (March 19, 1960), p. 34.

B.-EUDES, Marguerite, «les Cahiers Erta», Bulletin de la Société d'études et de conférences, vol. VI, no 3 (mars 1956), p. 78.

BALFOUR, Lisa, "A poet who paints or a painter who writes", The Montreal Star, vol. 99, no 17 (21 janvier 1967), p. 4.

BEAULIEU, Michel, «Faisceaux de la poésie québécoise», Presqu'Amérique, vol. I, no 2 (novembre-décembre 1971), p. 22-24 (p. 23).

BERNIER, Conrad, «Arts et lettres. Rencontre avec Roland Giguère», le Petit Journal, vol. 41, no 6 (semaine du 4 décembre 1966), p. 50-51.

BESSETTE, Gérard, «Le point de vue artistique», Canadian Literature, no 6 (Autumn 1960), p. 62-65.

BLOIS (pseudonyme), «Lauréat du grand Prix littéraire de la Ville de Montréal: Roland Giguère, poète, peintre et typographe», le Petit Journal, vol. XL, no 25 (17 avril 1966), p. 43-49. 
BORDELEAU, Jean-Marc, «Les mots enchantés de Roland Giguère», I'Information médicale et paramédicale, 4 avril 1978, p. 24.

BOUCHARD, C(harlemagne), «Roland Giguère ne colle pas à la poésie, c'est la poésie qui lui colle à l'âme», la Patrie, vol. 87, no 19 (semaine du 15 mai 1966), p. 67.

BOURASSA, André-G., Surréalisme et Littérature québécoise, (Montréal), Éditions l'Étincelle, (1977), 275 p. (v.p. 191-199).

«Le pouvoir du noir», Revue des Sciences humaines, (Lille), vol. XLV, no. 173 (janvier-mars 1979), p. 49-58.

BOURNEUF, Roland, «Roland Giguère», Europe, vol. 47, nos 478-479 (février-mars 1969), p. 157-163 (reproduit et traduit dans Ellipse, no 2 (Winter 1970), p. 30-37).

BRAULT, Jacques, «Roland Giguère, le poète de l'ébullition intérieure», Amérique française, vol. XVIII, no 2 (juin 1955), p. 132-139.

p. 41-45. , "Une poésie du risque...», Culture vivante, no 1 (1966), "Carnets d'un apprenti (extraits)», Liberté, vol. 21, no 2 (mars-avril 1979), p. 32-41.

CHAMBERLAND, Paul, «Nous ne sommes pas au monde: Giguère, Péloquin», Parti pris, vol. 3, no 7 (février 1966), p. 59-61.

, «Fondation du territoire», Parti pris, vol. 4, nos 9-12 (maiaoût 1967), p. 11-42 (v.p. 23-24, 26-31).

CHANEL-MALENFANT, Paul, la Partie et le Tout. Parcours de lecture chez Fernand Ouellette et Roland Giguère, Québec, les Presses de l'université Laval, 1983, 397 p. (v.p. 209-371).

CIMON, Renée (pseudonyme de Madeleine Bellemare), «Une poésie exemplaire, celle de Roland Giguère, l'Âge de la parole», le Carabin, vol. XXV.III, no 49 (30 mars 1967), p. 11.

, «Bibliographie de Roland Giguère», la Barre du jour, nos 11-13 (décembre 1967-mai 1968), p. 173-196.

CLOUTIER, Cécile, "La jeune poésie au Canada français», Incidences, no 7 (janvier 1965), p. 4-11.

COLLIN, W.E., "Letters in Canada. 1956. Publications in French», University of Toronto Quarterly, vol. XXV, no 3 (April 1956), p. 380-389 (v.p. 389-390).

DIESENDORF, Margaret, "Terre de Québec, Mère Courage», PoetryAustralia, no 16 (juin 1967), p. 56-59.

DOSTIE, Gaëtan, «En toutes lettres. La poésie québécoise. Le monde lumineux de Roland Giguère», le Jour, vol. I, no 27 (30 mars 1974), supplément, p. 2. 
En collaboration, «Connaissance de Giguère», numéro spécial de la Barre du jour, nọ 11-13 (décembre 1967-mai 1968), 196 p.

(Textes de Michel Beaulieu, Paul Chamberland, Renée Cimon, Claude Gauvreau, Gilles Hénault, Paul-Marie Lapointe, Gilles Marcotte, Gaston Miron, Jan Stafford).

FISETTE, Jean, le Texte automatiste. Essai de théorie/pratique de sémiotique textuelle, Montréal, les Presses de l'Université du Québec, 1977, XXIV, 181(1) p. (v.p. 103-129).

GAUVREAU, Claude, «Les livres de la muse. Roland Giguère, poète du Nouveau-Monde», le Haut-Parleur, vol. II, no 30 (28 juillet 1951), p. 5.

, "Documents. Les affinités surréalistes de Roland Giguère», Études littéraires, vol. V, no 3 (décembre 1972), p. 501-511.

GAY, Paul, «La jeune poésie», le Droit, vol. 56, no 74 (22 juin 1968), p. 8.

GIGUËRE, Richard, «D'un équilibre impondérable à une violence élémentaire. Evolution thématique de la poésie québécoise 1935-1965: Saint-Denys Garneau, Anne Hébert, Roland Giguère et Paul Chamberland», Voix et Images du pays, (1973), p. 51-90.

GLADU, Paul, "Quelques notes sur Roland Giguère», Notre temps, vol. XIII, no 8 (7 décembre 1957), p. 4.

GODIN, Gérald, «Les arts et les autres. Une interview de Gérald Godin avec Roland Giguère. Poète et amateur de hockey», le Magazine Maclean, vol. VI, no 12 (décembre 1966), p. 65-66.

, «Le Québec en mots dit. La poésie en 1968: quelques réflexions à Roland Giguère», le Jour, vol. V, nos 8-9 (été 1968), p. 75.

GRANDPRE, Pierre de, «Du 'Refus Global' à une poésie de l'appartenance. Roland Giguère», Histoire de la littérature française du Québec, tome III, Montréal, Librairie Beauchemin Limitée, 1969, 407 p. (v.p. 218-229).

HAMEL, Réginald, John HARE et Paul WYCZYNSKI, «Roland Giguère», Dictionnaire pratique des auteurs québécois, Montréal, Fides, 1979, p. 297-298.

HAMELIN, Jean, «Pierre Emmanuel et notre jeune poésie. La révolte a un temps: il faut maintenant qu'il y ait une, conquête», le Devoir, vol. L, no 79 (6 avril 1959), p. 9.

HÉBERT, François, «Roland Giguère», Liberté, vol. 21, no 2 (mars-avril 1979), p. 129-147.

«Les falaises de l'oeil», Liberté, vol. 24, no 139 (janvierfévrier 1982), p. 34-37.

HÉNAULT, Gilles, «Au début des années cinquante. Erta, arts graphiques et poésie», Vie des arts, vol. 22, no 90 (printemps 1978), p. 20-23. 
JASMIN, Claude, «L'instinct, la spontanéité, ça ne suffit pas! Roland Giguère», supplément à la Presse, vol. LXXIX, no 30 (17 novembre 1962), p. 23.

KATTAN, Naïm, «Lettre de Montréal: une saison abondante», Canadian Literature, no 28 (Spring 1966), p. 52-55. (v.p. 52-54).

LACÔTE, René, «La chronique de poésie de René Lacôte. Roland Giguère», les Lettres françaises, no 1241 (du 17 au 23 juillet 1968), p. 12 (reproduit dans le Devoir sous le titre «René Lacôte rend hommage au poète Roland Giguère», vol. LIX, no 199 (24 août 1968), p. 9.)

LAJOIE, Yvan, «Roland Giguère, à la recherche de l'essentiel», Études littéraires, vol. V, no 3 (décembre 1972), p. 411-428.

LAMY, Laurent, «Parmi nous la présence discrète et rayonnante de Roland Giguère», Forces, no 20 (1972), p. 46.

LAROCHE, Maximilien, «Notes sur le style de trois poètes: Roland Giguère, Gatien Lapointe et Paul Chamberland", les Cahiers de SainteMarie (Voix et Images du pays II), no 15 (mai 1969), p. 91-106.

LEMOINE, Wilfrid, «Les lettres. Un jeune poète montréalais imprime ses livres. La belle aventure des Éditions Erta», l'Autorité, vol. XXXIX, no 34, (30 janvier 1954), p. 6.

p. 58.

, «Roland Giguère. Le Poète», Vie des arts, no 9 (Noël 1957),

LE POITEVIN, Rémi, «Bellefleur et Giguère: la vie d'artiste est une chose sérieuse", Actualité, vol. 2, no 8 (août 1961), p. 10-11.

LOCKQUELL, Clément, «Influences qui ont affecté la poésie canadiennefrançaise contemporaine», Mémoires de la Société Royale du Canada, LVI, troisième série (juin 1962), p. 43-46 (v.p. 45).

MAILHOT, Laurent et Pierre NEPVEU, «Roland Giguère», la Poésie québécoise des origines à nos jours. Anthologie, Québec et Montréal, les Presses de l'Université du Québec et les Éditions de l'Hexagone, 1980, p. 364-373.

MARCOTTE, Gilles, «Roland Giguère», Mercure de France, no 1137 (mai 1958), p. 21.

, «Une poésie d'exil», Mercure de France, no 1137 (mai 1958), p. 5-9 (reproduit dans Canadian Literature, no 2 (Autumn 1959), p. 32-36, et dans Une littérature qui se fait, Montréal, HMH, 1962, p. 65-70.)

, "Connaissez-vous Roland Giguère?», Cité Libre, vol. XIII, no 45 (mars 1962), p. 26-28 (reproduit dans Une littérature qui se fait, Montréal, $\mathrm{HMH}, 1962$, p. 284-293.)

, «Roland Giguère à l'âge de la parole», la Presse, vol. LXXXI,

no 293 (18 décembre 1965), p. 1-3. 
, «Déflagrations à partir du surréalisme. Roland Giguère», le Temps des poètes, Montréal, HMH, 1969, p. 76-85.

MARTEAU, Robert, "L'atelier de Roland Giguère», Vie des arts, no $7 \overline{5}$ (été 1974), p. 51-54.

MAUGEY, Axel, «Roland Giguère», Poésie et Société au Québec (1937-1970), Québec, les Presses de l’Université Laval, 1972, p. 145-164.

MÉTIVIER, Henri, "Connaissance de Giguère», l'Action nationale, vol. LVIII, no 4 (décembre 1968), p. 389-391.

MICHAUD, Guildor, J., "L'Âge de la parole», Ratures, vol. I, no 1 (mars 1968), p. 32-37.

NOVELLI, Novella, Introduction à l'Età della parola, p. 7-11.

PALLASCIO-MORIN, Ernest, "Écrivains d'ici. Roland Giguère», l'Information médicale et paramédicale, 20 décembre 1977, p. 20.

PARADIS, Suzanne, "Connaissance personnelle du poète Roland Giguère», le Soleil, vol. 71, no 138 (8 juin 1968), p. 42.

PURDY, Anthony, «Roland Giguère et l'espace de l'autre: commentaire de deux poèmes», Voix et Images, vol. IV, no 2 (décembre 1978), p. 217-232.

RANCOURT, Jacques, «Au centre du feu. Roland Giguère» dans Serge Brindeau (éditeur), la Poésie contemporaine de langue française depuis 1945, Paris, Éditions Saint-Germain-des-Prés/Bordas, 1973, p. 571-577. , «Roland Giguère», «Poètes de l'identité québécoise», Poésie $I$, no 96-98 (mars-avril 1982), p. 59-68.

RICARD, François, «Giguère et Ducharme revisited», Liberté, vol. 16, no 91 (janvier-février 1974), p. 94-105.

ROBERT, Guy, «Roland Giguère», le Maclean, vol. II, no 7 (juillet 1971), p. 40 .

ROBILLARD, Jean-Paul, «Interview-éclair avec Roland Giguère», le Petit Journal, vol. 30 (6 novembre 1955), p. 63. , «Imprimeur mécène qui lance nos jeunes poètes cassés», le Petit Journal, vol. XXX, no 20 (11 mars 1956), p. 56.

ROYER, Jean, "Roland Giguère à l'âge de l'image», l'Action, vol. 59, no 17, 642 (23 avril 1966), p. 16.

, «La nuit en ce miroir est sans âge», l'Action, vol. LIX, no 17, 811 (12 novembre 1966), p. 13.

"Les rencontres internationales de Lurs. Graphisme et poésie au Québec», le Soleil, vol. LXXIX, no 301 (20 décembre 1975), p. D-9. 
«Roland Giguère. Prix Paul-Émile Borduas. Il faut voir audelà, prendre tout pour une fenêtre», le Devoir, vol. LXXII, no 275 (27 novembre 1982), p. 21-40.

SAINT-PIERRE, Marcel, "Le pouvoir du noir», le Quartier latin, vol. XLIX, no 15 (3 novembre 1966), supplément le Nouveau cahier, p. 4-5.

, «Connaissance de Giguère», la Barre du jour, nos 11-13 (décembre 1967-mai 1968), p. 5-8.

SARRAZIN, Jean, «Roland Giguère. À quoi mènent les arts graphiques...», la Presse, vol. LXXVI, no 133 (19 mars 1960), p. 35.

SYLVESTRE, Guy, Coron BRANDON et Carl F. KLINCK, Écrivains canadiens-Canadian Writers, Dictionnaire biographique. Édition revisée et augmentée, Montréal, HMH, 1966, p. 58-59.

SYLVESTRE, Guy, Panorama des lettres canadiennes-françaises, Québec, Ministère des Affaires culturelles du Québec, 1964, p. 52, 53, 57-59.

Union des écrivains québécois, «Roland Giguère», Dictionnaire des écrivains québécois contemporains, Montréal, Québec/Amérique, 1983, 399 p. (v.p. 178-179).

VAN SCHENDEL, Michel, «Poésie québécoise 1960-1965: l'apprivoisement du vertige ou la rencontre des nouvelles traditions», Livres et Auteurs canadiens, 1963, p. 13-22 (v.p. 16-18); dans Gilles Marcotte (compilateur), Présence de la critique, p. 195-196.

VIAU, Guy, «Paroles de peintre, Roland Giguère», Cahiers d'essai, no 1 (janvier 1958), p. 18-21.

VILLENEUVE, Pâquerette, «Une soirée poétique canadienne à Paris», le Devoir, vol. LII, no 2 (7 janvier 1961), p. 10.

\section{THÈSES}

CIMON, Renée (pseudonyme de Madeleine Bellemare), "Lecture de Roland Giguère», mémoire de licence ès lettres, Québec, Université Laval, $1967,59 \mathrm{f}$.

CHAMBLAS, Françoise, «Éluard et Giguère», thèse de maîtrise ès arts, Montréal, Université de Montréal, 1970, $116 \mathrm{f}$.

CHANEL-MALENFANT, Paul, «La partie et le tout; parcours de lecture chez Fernand Ouellette et Roland Giguère», thèse de doctorat ès lettres, Québec, Université Laval, 1979, X, 406-(21)f. (v.p. 223-390).

DUBOIS, Michelle, «Le 'Je' de l'énonciation dans l'Âge de la parole de Roland Giguère», thèse de maîtrise ès arts, Moncton, Université de Moncton, 1980 , IV-185 $\mathrm{f}$. 
GAGNIER-MORIN, Marie, «Roland Giguère où l'espace habité. Lecture phénoménologique de l'Âge de la parole et de la Main au feù, thèse de maîtrise ès arts, Québec, Université Laval, 1981, IX, 77 f.

GAUTHIER, Judith, «L'expérience de la création littéraire dans la poésie de Roland Giguère», thèse de maîtrise ès arts, Montréal, Université McGill, 1975, IV-169, III f.

LAJOIE, Yvan, "Cinq poèmes de Roland Giguère. Une lecture en fonction des symboles de la vie et de la mort», thèse de maîtrise ès arts, Québec, Université Laval, 1972, XIV, $146 \mathrm{f}$.

MESSIER, Marcel, «Considérations sur les structures du langage poétique: lecture analytique d'un texte de Roland Giguère», thèse de maîtrise ès arts, Québec, Université Laval, 1981, (iii), 108, (1i) f.

PRONOVOST, Jean-Guy, «L'itinéraire des couleurs chez Roland Giguère», thèse de maîtrise ès arts, Trois-Rivières, Université du Québec à Trois-Rivières, 1975, $233 \mathrm{f}$.

\section{ÉTUDES PARTICULIĖRES}

\section{Adorable femme des neiges (Noir aussi l'Âge de la parole)}

(Anonyme), «Un poème qui coûte $\$ 125$. !, le Devoir, vol. LI, no 83 (16 avril 1960), p. 11 .

BROCHU, André, Dictionnaire des oeuvres littéraires du Québec, tome III, p. 11-14.

\section{Les Armes blanches (Noir aussi l'Âge de la parole)}

BROCHU, André, «Commentaire de 'Roses et ronces'», la Barre du jour, nos 11-13 (mai 1968), p. 45-64 (reproduit dans l'Instance critique 1961-1973, Montréal, Leméac, 1974, p. 275-308).

CARTIER, Georges, «Études critiques. Les Armes blanches», Lectures, tome X, no 10 (juin 1954); p. 433-435.

DUHAMEL, Roger, «Un document spirituel capital dans notre littérature», la Patrie (du dimanche), vol. XX, no 23 (6 juin 1954), p. 83.

GERVAIS, André, «Lecture/écriture de Roland Giguère: les Mots-flots», Voix et Images, vol. III, no 2 (décembre 1979), p. 302-319.

HAEFFELY, Claude, «Notes sur Armes blanches», Amérique française, vol. XII, no 1 (avril 1954), p. 70-73.

LAROCHE, Maximilien, «Sur un poème de Roland Giguère», l'Action nationale, vol. 56, no 9 (mai 1967), p. 920-925.

LAROCHE, Maximilien, Dictionnaire des oeuvres littéraires du Québec, tome III, p. 64-68. 
LEMOINE, Wilfrid, «Avec ses Armes blanches, Roland Giguère invente un univers d'éblouissements», l'Autorité, vol. XXXIX, no 31 (3 avril 1954), p. 6.

LOCKQUELL, Clément, "Chronique des livres. Le Dit de l'enfant mort, de Gabriel Charpentier; les Armes blanches de Roland Giguère, le Soleil, vol. LXXIII, no 112 (8 mai 1954), p. 4.

MARCOTTE, Gilles, «Vie des lettres. Une poésie du bout du monde. Les Armes blanches, par Roland Giguère», le Devoir, vol. XLV, no 77 (3 avril 1954), p. 6.

VALOIS, Marcel (pseudonyme de Jean Dufresne), «Arts et Lettres. De poète en poète", la Presse, vol. LXX, nó 166 (1er mai 1954), p. 74.

\section{Le Défaut des ruines est d'avoir des habitants Noir aussi l'Âge de la parole, la Main au feu et Forêt vierge folle)}

(Anonyme), «les Lettres et les Arts. Oeuvres graphiques et présentation de poèmes par Roland Giguère», la Presse, vol. LXXIV, no 5 (19 octobre 1951), p. 74.

GRANDPRÉ, Pierre de, «La vie des lettres. Le Défaut des ruines est d'avoir des habitants", le Devoir, vol XLVIII, no 287 (7 décembre 1957), p. 11.

HAECK, Philippe, "La muraille de Chine», Naissances. De l'écriture québécoise, Montréal, VLB éditeur, 1979, p. 68-73.

LEMOINE, Wilfrid, «Livres. Le Défaut des ruines est d'avoir des habitants...», Vie des arts, no 9 (Noël 1957), p. 41.

MAILLET, Andrée, «Le feuilleton d'Andrée Maillet. Le phénomène Roland Giguère», le Petit Journal, vol. XXXII, no 53 (27 octobre 1957), p. 84.

PONTBRIAND, Jean-Noël et Roger CHAMBERLAND, Dictionnaire des oeuvres littéraires du Québec, tome III, p. 267-271.

SAINT-MARTIN, F(ernande), "Arts et Lettres. Le poète d'une génération», la Presse, vol. LXXIV, no 17 (2 novembre 1957), p. 74. , «La poésie. Des mots sur notre silence», la Presse, vol. LXXV, no 202 (13 juin 1959), p. 55.

SAINTE-MARIE, Micheline, "Qui est 'Miror'. Une étude sur Roland Giguère», Présence, décembre 1957-janvier 1958, p. 7, 10.

SYLVESTRE, Guy, «Livres en français. La poésie», University of Toronto Quarterly, XXVII, no 4 (July 1958), p. 542-552 (v.p. 551).

\section{Faire naître}

BONENFANT, Joseph, Dictionnaire des oeuvres littéraires du Québec, tome III, p. 362-364. 


\section{Images apprivoisées}

PONTBRIAND, Jean-Noël et Roger CHAMBERLAND, Dictionnaire des oeuvres littéraires du Québec, tome II, p. 267-271.

LEMOINE, Wilfrid, «Deux poètes qui ne craignent pas le coup de dés. François Dodat (Français). Roland Giguère (Canadien)», l'Autorité, vol. XXXIX, no 27 (23 janvier 1954), p. 6.

MARCOTTE, Gilles, «Vie des lettres. Poèmes pour le plaisir. Éloi de Grandmont. Gilles Hénault. Roland Giguère», le Devoir, vol. XLV, no 17 (23 janvier 1954), p. 6 .

\section{Midi perdu (Voir aussi l'Āge de la parole)}

CHAMPAGNE, Guy, Dictionnaire des oeuvres littéraires du Québec, tome III, p. 1023-1024.

M(AILLET), A(ndrée), «Revue des livres. Midi perdu», Amérique française, vol. XI, no 5 (novembre 1953), p. 76-77.

MARCHAND, Jacques, «Roland Giguère», Claude Gauvreau, poète et mythocrate, Montréal, VLB éditeur, 1979, p. 241-247.

\section{Les Nuits abat-jour (Noir aussi l'Âge de la parole)}

CHAMPAGNE, Guy, Dictionnaire des oeuvres littéraires du Québec, tome III, p. 1022-1024.

\section{3 pas}

CHAMPAGNE, Guy, Dictionnaire des oeuvres littéraires du Québec, tome III, p. 1022-1024.

\section{Yeux fixes ou l'ébullition de l'intérieur (Noir aussi l'Âge de la parole)}

AUBIN, Jean-Pierre, «Roland Giguère: l'Âge de la parole. Yeux fixes», le Quartier latin (supplément), vol. XLVIII, no 26 (27 janvier 1966), p. 6.

BERTRAND, Claude, «Lecture des yeux fixes», la Barre du jour, nos 11-13 (décembre 1967-mai 1968), p. 11-20.

FISETTE, Jean, le Texte automatiste. Essai de théorie/pratique de sémiotique textuelle, Montréal, les Presses de l'Université du Québec, 1977, XXIV, p. 109-129.

HAECK, Philippe, «L'ébullition de l'intérieur. La mariée n'est plus à elle», Naissances. De l'écriture québécoise, Montréal, VLB éditeur, 1979, p. 41-51. PARADIS, Suzanne, Dictionnaire des oeuvres littéraires du Québec, tome III, p. 1078-1089.

\section{L'Âge de la parole}

(Anonyme), «Rétrospective», le Quartier latin, vol. II, no 11 (13 janvier 1966), p. 2. 
, «l'Âge de la parole», la Patrie, vol. 87, no 5 (6 février 1966), p. 52. , «le Grand Prix de la Ville de Montréal va à Roland Giguère», le Devoir, vol. LVII, no 80 (6 avril 1966), p. 3.

" «Décerné à Paris. Le prix France-Canada à Roland Giguère», le Devoir, vol. LVII, no 274 (25 novembre 1966) p. 12.

, «Roland Giguère reçoit le prix France-Canada 1966», le Soleil, vol. 69, no 280 (25 novembre 1966), p. 20.

, «Sur la poésie de Roland Giguère», le Nouvelliste, vol. 48, no 111 (11 mars 1968), p. 8.

, «\$3,000 for best entry. Roland Giguère awarded city's literature prize», The Montreal Star, vol. 98, no 81 (6 avril 1966), p. 21.

, «Fiche J.L.Q. L'Âge de la parole», Vient de paraître, vol. 4, no 4 (septembre 1968), p. 38.

AUBIN, Jean-Pierre, "Roland Giguère: l'Âge de la parole», le Quartier latin, (supp.), vol. XLVIII, no 26 (27 janvier 1966), p. 6.

$\mathrm{A}(\mathrm{YLWIN}), \mathrm{U}($ lric), "Comptes rendus. Roland Giguère, l'Âge de la parole, Poèmes de 1949-1960", Études françaises, vol. III, no 4 (novembre 1967), p. $438-439$.

BERNIER, Thérèse, «Le Canada français a tenu la vedette dans le monde des arts», le Soleil, vol. 69, no 5 (3 janvier 1966), p. 9.

BERTRAND, André, «Une littérature en acte», le Quartier latin (supplément), vol. II, no 13 (27 janvier 1966), p. 1.

BLOIS (pseudonyme), «On présente tout Roland Giguère», le Petit Journal, vol. 40, no 8 (semaine du 19 décembre 1966), p. 35.

, "Laureat du grand prix litteraire de la Ville de Montreal. Roland Giguère, poète, peintre et typographe», le Petit Journal, (17 avril 1966), p. 42-43.

BOURNEUF, Roland, «Horizons littéraires. L'Âge de la parole. De Roland Giguère», le Soleil, vol. 69, no 93 (16 avril 1966), p. 27.

CHAMBERLAND, Paul, «Les Livres. Nous ne sommes pas au monde: Giguère, Péloquin», Parti pris, vol. 3, no 7 (février 1966), p. 59-61.

CIMON, Renée (pseudonyme de Madeleine Bellemare), «Une poésie exemplaire, celle de Roland Giguère. L'Âge de la parole», le Carabin, vol. XXVII; no 49 (30 mars 1967), p. 4.

DAIGNEAULT, Claude, «Dans les coulisses», le Soleil, vol. 69, no 93 (16 avril 1966), p. 24.

D(AIGNEAULT), C(laude), "Le monde imaginé de Roland Giguère», le Soleil, vol. 69, no 99 (23 avril 1966), p. 26. 
DUCIAUME, Jean-Marcel, Dictionnaire des oeuvres littéraires du Québec, tome IV (à paraître).

DUDEK, Louise, "Canadien Poet Roland Giguère one among the chosen», The Gazette, vol. 188 (April 16, 1966), p. 14.

GAY, Paul, «La jeune poésie», le Droit, vol. 56, no 74 (22 juin 1968), p. 8. KATTAN, Naïm, «Lettre de Montréal: une saison abondante», Canadian Literature, no 28 (Spring 1966), p. 52-55 (v.p. 52-54).

KATTAN, Naïm, «la Poésie», Bulletin du cercle juif, vol. 12, no 112 (avril 1966), p. 4.

LACÔTE, René, «Claire Lejeune. Roland Giguère», les Lettres françaises, no 1133 (du 26 mai au ler juin 1966), p. 8.

LANGEVIN, Lysianne, «La main du bourreau finit toujours par pourrir: analyse», la Barre du jour, nos 39-40-41 (printemps-été 1973), p. 206-226.

LE GRAND, Albert, «Une parole enfin libérée», Maintenant, nos 68-69 (15 septembre 1967), p. 267-272.

MARCHAND, Olivier, «La poésie revient, Villon!», la Presse, vol. LXXXIV, no 65 (16 mars 1968), p. 27.

MARCOTTE, Gilles, "Roland Giguère à l'Âge de la parole», la Presse (supplément littéraire), vol. LXXXI, no 293 (18 décembre 1965), p. 1, 3.

MONTBIZON, R., "Ả Montréal: le Concours artistique du Québec», Vie des arts, no 45 (hiver 1966-67), p. 60-61.

POISSON, Roch, «Vie littéraire», Photo-journal, vol. 29, no 40 (19 janvier 1966), p. 69.

SABBATH, Lawrence, «A Prize that's unique», Montréal' 66, vol. 3, no 7 (juillet 1966), p. 18-19.

SAINT-PIERRE, Marcel, «Livres. La poésie de Giguère, une arme à deux tranchants», Désormais, vol. 1, no 1 (avril 1966), p. 14.

SYLVESTRE, Guy, «Les livres de la semaine. La poésie C.-F. L'Âge de la parole de Roland Giguère», le Devoir, vol. LVI, no 301 (24 décembre 1965), p. 10 (reproduit dans Québec 66, vol. 6 (mai 1966), p. 81-83, et dans Livres et Auteurs canadiens, 1965, p. 77-78.

«Livres en français. La poésie», University of Toronto Quarterly, vol. XXXV, no 4 (July 1966), p. 503-509 (v.p. 503-505).

THÉBERGE, Jean-Yves, «À côté de Grandbois... Roland Giguère. L'Âge de la parole», le Canada français, vol. CVI, no 35 (20 janvier 1966), p. 26.

VIATTE, Auguste, «Chronique des lettres françaises hors de France: le Canada», Conjonction, no 105 (octobre 1967), p. 5-11. 


\section{Pouvoir du noir}

LAROCHE, Maximilien, «Pouvoir du noir de Roland Giguère ou la poésie comme critique de l'idéologie», dans En collaboration, Deux Études sur la poésie et l'idéologie québécoises, les Cahiers de l'ISSH, no 3 (deuxième trimestre 1975), p. 19-40.

\section{La Main au feu}

(Anonyme), «Ducharme, Giguère, Faucher. Prix du Gouverneur général», le Devoir, vol. LXV; no 114 (18 mai 1974), p. 11.

, «Prix littéraires pour trois Québécois», le Droit, vol. 62, no 45 (18 mai 1974), p. 20.

, «Roland Giguère refuse le prix du Gouverneur», le Devoir, vol. LXV, no 118 (23 mai 1974), p. 12.

, «Roland Giguère refuse le prix du Gouverneur!», Montréal-

Matin, vol. XLIV, no 322 (23 mai 1974), p. 31.

212 (juin 1974).

, «la Main au feu (1949-1968)», le Livre Canadien, vol. 5, no

BROCHU, André, «Roland Giguère. La Main au feu, Éditions de l'Hexagone», Livres et Auteurs québécois, 1973, p. 93-96.

GODBOUT, Jacques, «Les livres. Un portrait qui parle», le Maclean, vol. 13, no 12 (décembre 1973), p. 4, 6 (v.p. 4).

LEMIEUX, Jacques et J.-P. LÉGARÉ, «La poésie. Roland Giguère, les yeux fixés sur le Québec, livre... ses secrets», le Devoir, vol. LXV, no 132 (8 juin 1974), p. 16.

LEMIEUX, Jacques, «La poésie. Plus de patates que de perles», le Devoir, vol. LXV, no 132 (18 janvier 1975), p. 17.

MARCOTTE, Gilles, "La Main au feu», Études française, vol. 10, no 2 (mai 1974), p. 136.

POULIN, Gabrielle, «La Main au feu, de Roland Giguère», Relations, vol. 34, no 396 (septembre 1974), p. 251-253.

THÉBERGE, Jean-Yves, «Roland Giguère», le Canada français, vol. CXIV, no 35 (23 janvier 1974), p. 60.

\section{J'imagine}

DE ROUSSAN, Jacques, «Lectures. Une imagination inquiète», Vie des arts, vol. 21, no 85 (hiver 1976-1977), p. 92-97 (v.p. 93).

DOSTIE, Gaëtan, «Livres. Ce pays 'rapaillé' à travers Giguère et Lapointe», le Jour, vol. III, no 108 (9 juillet 1976), p. 18. 


\section{Forêt vierge folle}

BONENFANT, Yvon, «Forêt vierge folle», Nos Livres, vol. 10, no 215 (juin-juillet 1979).

GIGUERE, Richard, «Letters in Canada 1978. La poésie», University of Toronto Quarterly, vol. XLVIII, no 4 (Summer 1979), p. 354-362 (v.p. 354-355).

HAMBLET, Edwin, «La poésie québécoise», Canadian Literature, no 76 (spring 1978), p. 92-93.

LEMAIRE, Michel, «La poésie I. Multiple Giguère», Lettres québécoises, no 13 (février 1979), p. 17-18.

MARCHAND, Jacques, «Forêt vierge folle», Livres et Auteurs québécois, 1978, p. 121-123.

MARCOTTE, Gilles, "Prendre tout pour une fenêtre», le Devoir, vol. LXX, no 28 (3 février 1979), p. 17-21.

MARTEL, Réginald, «Roland Giguère, artisan de la splendeur», la Presse, vol. XCV, no 47 (24 février 1979), p. D-3.

«Littérature. Pour nous donner la lumière», la Presse, vol. XCV, no 47 (24 février 1979), p. D-3.

MARTIN, André, «Lecture. De l'Âge de la parole à l'âge de l'image», Vie des arts, vol: 24, no 97 (hiver 1979-1980), p. 84-89 (v.p. 89).

ROYER, Jean, «Pour célébrer Roland Giguère. La main libère la parole», le Devoir, vol. LXX, no 28 (3 février 1979), p. 17-19. 